Review

\title{
The Role of Innate Immunity in Conditioning Mosquito Susceptibility to West Nile Virus
}

\author{
Abhishek N. Prasad ${ }^{\dagger}$, Doug. E. Brackney ${ }^{\dagger}$ and Gregory D. Ebel * \\ Department of Microbiology, Immunology and Pathology, Colorado State University, Fort Collins, \\ CO 80523, USA; E-Mails: Abhi.Prasad@colostate.edu (A.N.P.); Doug.Brackney@colostate.edu (D.E.B.) \\ $\dagger$ These authors contributed equally to this work. \\ * Author to whom correspondence should be addressed; E-Mail: Gregory.Ebel@colostate.edu; \\ Tel.: +1-970-491-8374; Fax: +1-970-491-8707.
}

Received: 29 August 2013; in revised form: 13 November 2013/Accepted: 9 December 2013 / Published: 13 December 2013

\begin{abstract}
Arthropod-borne viruses (arboviruses) represent an emerging threat to human and livestock health globally. In particular, those transmitted by mosquitoes present the greatest challenges to disease control efforts. An understanding of the molecular basis for mosquito innate immunity to arbovirus infection is therefore critical to investigations regarding arbovirus evolution, virus-vector ecology, and mosquito vector competence. In this review, we discuss the current state of understanding regarding mosquito innate immunity to West Nile virus. We draw from the literature with respect to other virus-vector pairings to attempt to draw inferences to gaps in our knowledge about West Nile virus and relevant vectors.
\end{abstract}

Keywords: West Nile virus; innate immunity; RNAi; Toll; Imd; Jak/STAT; autophagy; apoptosis; arbovirus; mosquito

\section{Introduction}

Arthropod-borne viruses (arboviruses) perpetuate in nature in transmission cycles involving both vertebrate animals, including human beings, and hematophagous arthropods, mainly mosquitoes and ticks. The genus Flavivirus (family Flaviviridae), including dengue viruses 1-4 (DENV), yellow fever virus (YFV), tick-borne encephalitis virus (TBEV), Japanese encephalitis virus (JEV), West Nile virus 
(WNV) and many others is the most significant group of arboviruses. Several billion individuals, mainly residing in the tropics, are currently at risk of infection with one or more of these agents, and despite the availability of safe vaccines for some of them, several hundred thousand human infections occur annually. The relatively intractable public health burden imposed by arboviruses and the extreme difficulty in designing and implementing effective countermeasures against them stems in part from the complexity of transmission cycles that involve arthropod populations. Mosquito populations, for example, may be influenced by habitat and host availability, temperature and rainfall. Subtle fluctuations in any of these factors may have unpredictably dramatic impacts on the dynamics of virus replication in mosquitoes and human and animal disease [1-4]. Moreover, mosquitoes are clearly critical to the lifecycle and epidemiology of arboviruses, including WNV, the subject of this review.

Basic studies of the molecular mechanisms that underpin the host-virus interaction in WNV have proceeded vigorously in recent years. These studies, which aim to identify and characterize mechanisms that influence the dynamics of virus infection and contribute to clinical outcomes, have led to a greatly enhanced understanding of how hosts and viruses interact. For example, the importance of vertebrate innate immunity [5-7], T-, B-, and other lymphocytes [8-10] and complement [11-13] have been shown to influence WNV disease to varying degrees. Importantly, the most important vertebrate immune effectors are absent in mosquitoes. Therefore, despite the significance of mosquitoes to WNV perpetuation and transmission, comparatively little is known about the molecular mechanisms that influence the mosquito-WNV interaction. Studies of mosquito responses to WNV (and other arboviruses) are thus in their infancy.

Work on mosquito-arbovirus interactions has addressed two related points. First, studies have sought to characterize antiviral pathways activated in mosquitoes following virus infection and determine how they limit virus replication (i.e., how do they protect mosquitoes from lethal infection). Second, they have sought to understand how mosquito antivirus responses contribute to the likelihood that a given mosquito will become infected by a virus, support its replication and dissemination beyond the mosquito midgut (the site of initial infection) and ultimately transmit virus (i.e., the mosquito's "vector competence"). An important question that has not yet been fully explored is the extent to which these two related points (antiviral responses and vector competence) are linked. It has been more or less implicitly presumed that antiviral responses in mosquitoes ought to contribute in some way to the phenotype of vector competence. That this is true of naturally occurring mosquito vectors of arboviruses is far from clear: The literature demonstrates that vector competence is a quantitative genetic trait under the control of several loci that account for a surprisingly small proportion of the observed variation in vector competence [14-16]. A full accounting of the functional genomics and systems biology of the mosquito antivirus response is required in order to push forward our current understanding of vector competence.

Accordingly, this review addresses the current literature surrounding mosquito antivirus responses, with a particular focus on Culex mosquitoes and WNV. Specifically, we cover recent literature on small RNA regulatory pathways (siRNA, piRNA and others), antiviral signaling cascades (Toll, Jak-STAT, etc.), and the cellular processes of autophagy and apoptosis. Figure 1 has been included to illustrate each of the pathways discussed in this review. Within this review, we draw heavily on the literature generated in related systems, including Aedes mosquitoes and the model organism Drosophila melanogaster. This tendency reflects a critical shortcoming in the current state of the field: 
Very little work on the molecular mechanisms that influence virus infection in mosquitoes has been performed using appropriate virus-vector pairs. Nonetheless, we believe that studies conducted using related systems and model organisms provide critical insights, and the way forward for investigators focusing on arbovirus-mosquito interactions. We conclude with remarks intended to summarize the state of knowledge and identify key areas for future research.

\section{Small RNA Regulatory Pathways}

Small RNA regulatory pathways (SRRPs) are an integral component of endogenous pre- and post-transcriptional gene regulation. Three primary classes of small RNAs exist within metazoans: micro-RNAs, (miRNAs), small-interfering RNAs (siRNAs), and PIWI-interacting RNAs (piRNAs), being distinguished by both the size of the small RNA product, and their biogenesis. Invertebrates lack type I and type III interferon (IFN) responses, which are the main innate immune pathway through which vertebrates respond to virus infection. Rather, in invertebrates, there is ample evidence highlighting the role of SRRPs in antiviral innate immunity. Exogenous RNA interference (exo-RNAi) via the siRNA pathway appears to be the primary small RNA response; however, involvement of the piRNA pathway in antiviral defense has recently been described in both mosquitoes and mosquito cell culture. In this section, we will discuss the role of these pathways and their components in the context of antiviral defense to WNV.

Figure 1. Mosquito innate immune pathways. (A) Toll-pathway signaling is initiated by Spätzle 5 binding to the Toll receptor, initiating the dissociation of Dif from Cactus. Dif translocates to the nucleus where it initiates transcription of antimicrobial genes; (B) Recognition of pathogen-associated molecular patterns (PAMPs) by peptidoglycan recognition protein-LC results in activation and translocation of Relish to the nucleus, and subsequent transcription of antimicrobial genes; (C) Extracellular unpaired (upd) binds to the transmembrane receptor Domeless, initiating dimerization of Stat92E, resulting in the subsequent transcription of antimicrobial genes. (D) The prophenoloxidase cascade is activated by PAMP activation of the serine proteinase cascade. The pro-prophenoloxidase activating enzyme (PPAE) peptide becomes activated upon cleavage by upstream serine proteinases, resulting in the activation of phenoloxidase. Phenoloxidase converts phenol to quinones, and eventually melanin; (E) The piRNA pathway may play a compensatory role to the exo-RNAi pathway during arbovirus infection. Primary piRNAs are fed into the "ping-pong dependent" amplification cycle, whereby cleavage of cognate mRNA leads to production of secondary piRNAs. These in turn bind to complementary transcripts, re-starting the cycle. (F) The exo-RNAi pathway is initiated by cellular recognition of exogenous dsRNA. DCR2 cleavage of long dsRNA results in 21nt siRNA duplexes, which are loaded into the RISC. Single-stranded guide strand siRNAs direct RISC to viral genomic RNA by sequence complementarity, leading to cleavage of the virus RNA by AGO2. The $\mathrm{DExD} / \mathrm{H}$-domain of DCR2 also leads to induction of the secreted peptide vago, activating Jak/STAT and leading to downstream activation of the virus-inducible gene vir-1, restricting virus replication. (G) Signaling by initiator caspases to effector caspases results in programmed cell death. H) Recognition of PAMPs by unknown PRRs inhibits TOR-mediated negative 
regulation of phagophore formation, leading to mature autophagosomes, which fuse to lysosomes leading to degradation of the contents of the autophagosomes.

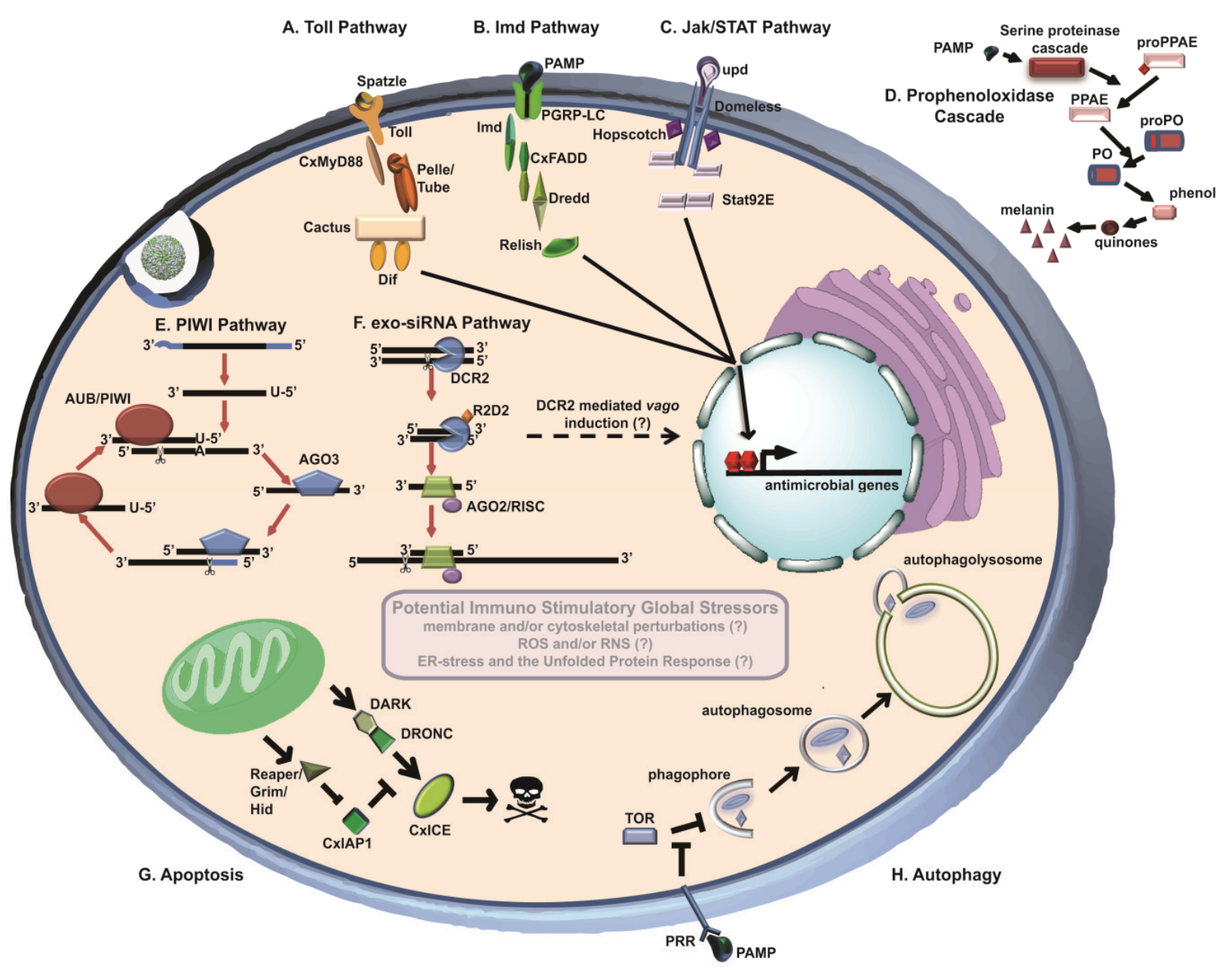

\subsection{Exo-siRNA Pathway}

RNA interference (RNAi) was first described in plants as a mechanism for "post-transcriptional gene silencing" [17], and later, "virus-induced gene silencing" [18], two phenomena which, at the time, were seemingly unrelated. Several years after these initial observations, double-stranded RNA (dsRNA) was found to be the trigger for RNAi in Caenorhabditis elegans [19] and Drosophila melanogaster [20]. In invertebrates and plants, exo-RNAi is induced by cellular recognition of long dsRNAs as pathogen-associated molecular patterns (PAMPs), which naturally occur as viral genome replication intermediates and genomic RNA secondary structures in the case of RNA viruses, and as convergent transcripts in DNA viruses. These dsRNAs are recognized and cleaved by Dicer-2 (DCR2), a cytoplasmic RNase III enzyme, resulting in 19-23 base pair (bp) fragments (predominately 21 bps) termed siRNAs. siRNA duplexes produced in this manner exhibit 5' monophosphates and 3' hydroxyls, as well as two-nucleotide (nt) overhangs on their $3^{\prime}$ termini. These siRNAs are then loaded into the Argonaute-2 (AGO2)-containing RNA-induced silencing complex (RISC) through association with a DCR2/R2D2 heterodimer [21]. After the duplex is unwound a single-stranded RNA known as the guide strand remains associated with the RISC, and is 2'-O methylated by the methyltransferase 
DmHEN1 [22,23], and the complimentary strand, known as the passenger strand, is discarded. The RISC then recognizes cognate mRNA (in this case, virus genomic RNA) by sequence complementarity with the guide strand. Degradation of the target occurs through the Slicer endonuclease activity of AGO2 [24]. Unlike miRNAs, where mismatches between the guide strand and target are tolerated, even a single mismatch in complementarity between a siRNA and its target can result in diminished or abolished silencing $[25,26]$. In this way, the siRNA pathway acts as a highly potent antiviral pathway in controlling arbovirus infection.

The role of the siRNA pathway in antiviral defense in arthropods has been the subject of intense investigation in recent years. In Drosophila, numerous studies have demonstrated that RNAi inhibits virus replication [27-29]. Notably, Drosophila with a null mutant DCR2 enzyme exhibit $\sim 70 \%$ mortality and dramatically higher virus titers when inoculated with Sindbis virus (SINV, Togaviridae), as compared to wild-type controls [28]. In mosquitoes, evidence of the involvement of the siRNA pathway during arbovirus infection has been observed in several virus/arthropod pairings, including o'nyong-nyong virus (ONNV, Togaviridae) in Anopheles gambiae [30], SINV in Aedes aegypti [31], and DENV in Ae. aegypti [32]. viRNAs produced in response to WNV had been detected in Drosophila S2 cells, but not Ae. albopictus C6/36 cells [33] due to a dysfunctional siRNA pathway [34] resulting from a single nucleotide deletion introducing a premature stop codon within the open reading frame (ORF) of DCR-2 [35]. One potential pitfall in interpreting the results of these studies is the utilization of non-natural virus/vector pairings and/or infection routes (i.e., intrathoracic inoculation), with the exception of Sanchez-Vargas et al. [32]. Brackney et al. utilized next-generation sequencing (NGS) to profile the antiviral RNAi response to WNV in its natural vector, Cx. quinquefasciatus mosquitoes, following peroral infection, and found viRNAs produced in the midgut of mosquitoes at 7 and 14 days post-infection (dpi) [36]. viRNAs produced in this manner were primarily $21 \mathrm{nts}$ in length (indicative of DCR2 processing), and were asymmetrically distributed along the length of the virus genome.

Given the requirement for high target sequence complementarity in siRNAs, it comes as no surprise that RNAi can drive viral diversity and evolution through the generation of RNAi-escape mutants that differ sufficiently from the master sequence. Viral escape from one or a few transfected siRNAs has been observed in a variety of different systems, including hepatitis C virus (HCV, Flaviviridae) [37], human immunodeficiency virus-1 (HIV-1, Retroviridae) [26], turnip mosaic virus (TuMV, Potyviridae) [38], and poliovirus (PV, Picornaviridae) [39]. Based on the observation that WNV population structure was more complex in mosquitoes than in birds [40], it was hypothesized that the mosquito RNAi pathway may serve as a potent selective pressure on the virus to favor generation and maintenance of rare mutants. Indeed, a correlation between nucleotide targeting and increased likelihood for corresponding point mutations has been observed [36], though it is important to note that this observation was associative, and does not demonstrate causation. Taken together, these studies highlight the role this pathway may play in mosquito innate antiviral immunity, and shed light on how it may influence virus diversification and evolution. Moreover, the error rate of the virus replicase complex may serve in part as an evolutionary mechanism for circumventing the mosquito siRNA-based antiviral response through the generation of rare mutants which differ sufficiently from the master sequence. This is circumstantially supported by the observation that a chikungunya virus (CHIKV, Togaviridae) mutant expressing a high fidelity RNA-dependent RNA polymerase (RdRP) exhibited 
lower infection and dissemination titers in mosquitoes as compared to the wild-type virus [41]. However, it should be noted that the molecular basis for this observation was not investigated, and that immunological factors other than RNAi could potentially influence the fitness of a genetically homogenous virus population.

Systemic RNAi, first described in plants and worms, is the process by which the siRNA response spreads beyond the site of initiation into surrounding cells and tissues (see [42] for a review). The mechanism of spread differs between plants and animals, with short-distance transport of siRNAs in plants occurring through plasmodesmal junctions connecting cells, and long-distance transport being mediated by the vascular system. In C. elegans, spread of the RNAi signal is mediated by members of the SID family of transmembrane transporters $[43,44]$. Evidence suggests that this process may occur in dipterans as well, and has broad implications in understanding RNAi-based antiviral immunity in these systems. Studies in flies have revealed a systemic RNAi pathway [45,46], and cell-to-cell spread of viRNAs produced in response to Semliki Forest virus (SFV, Togaviridae) infection has been demonstrated in mosquito cell culture [47]. In C. elegans, primary viRNAs are amplified in a target dependent manner by the endogenous RdRP RRF-1, which contributes to systemic RNAi spread and subsequent maintenance of long-term silencing [48]. Secondary viRNAs produced in this manner are composed entirely of antisense polarity, and exhibit 5' di- or triphosphates, making them structurally unique, and thus a distinct class of small RNA molecules [49,50]. While an RdRP capable of amplifying viRNAs has not been conclusively identified in dipterans, a recent publication suggests that viral RNA produced during flock house virus (FHV, Nodaviridae) in Drosophila can be reverse-transcribed into viral cDNAs mediated by the reverse transcriptase activity of endogenous long terminal repeat (LTR)-retrotransposons [51]. Viral cDNA produced in this manner may then be integrated into the host cell genome, or circularized into stable, extrachromasomal DNA which can be efficiently transcribed into dsRNAs that can be fed back into the siRNA pathway, leading to a primed immune response, and allowing for a persistent infection to develop. Additionally, viRNAs produced in response FHV infection in C. elegans have been observed to be transgenerationally inherited from mother to offspring in successive generations [52], raising the intriguing possibility that similar mechanisms of amplification and non-Mendelian, extrachromosomal inheritance of small RNAs may exist in mosquitoes as well, though it should be noted that to date, there is a lack of experimental data supporting this. Given the aforementioned importance of the RNAi pathway in mosquito innate immunity to viral infection, inheritance of viRNAs might be expected to influence mosquito vector competence and arbovirus populations in nature.

\subsection{Vago}

Cross-talk between SRRPs and other innate immune pathways is an emerging feature of mosquito antiviral defense against arboviruses. DCR2 belongs to the same family of DExD/H-box helicases as the RIG-I-like receptors, which are involved in the induction of the IFN response in mammalian systems. Deddouche et al. reported that the secreted peptide vago is induced in D. melanogaster in response to Drosophila C virus (DCV, Dicistroviridae) and SINV infection in the fat bodies of flies, and that induction of vago was reliant on the amino terminal DExD/H-box domain of DCR2 [53]. Notably, infection by FHV did not induce vago expression, likely due to the FHV encoded viral suppressor of RNAi, B2, which binds dsRNA thereby interfering with downstream signaling by 
DCR2. While vago was shown to control virus infection in this study, the mechanism by which it did so was unclear. Pradakar et al. further explored vago's role in antiviral immunity using cultured mosquito cells and WNV [54]. It was found that vago was effective in limiting WNV infection in Cx. quinquefasciatus-derived Hsu cells, and that induction of vago resulted in activation of Jak/STAT signaling, leading to the induction of the STAT-dependent virus inducible gene vir-1, thereby restricting WNV replication. Although the identity of the cellular receptor for vago is currently not clear, studies published to date suggest that components of the RNAi pathway can have diverse, multifunctional roles in controlling arbovirus infections in mosquitoes.

\subsection{PIWI-Interacting RNA Pathway}

Recent evidence suggests that a second class of small RNAs with distinct biogenesis may also be induced in arthropods following virus infection. The p-element induced wimpy testes (PIWI) class of Argonaute proteins were first discovered in 1997 by Lin et al. and shown to be potent regulators of spermatogenesis in Drosophila [55]. In 2006, several studies were published indicating that PIWI proteins interact with a unique class of small RNAs, named PIWI-interacting RNAs (piRNAs; the nomenclature at the time was variable, and they were also referred to as repeat-associated small interfering RNAs [rasiRNA], which are now considered a distinct subclass of piRNAs) [56-60]. piRNAs exhibit some unique features that distinguish them from miRNAs and siRNAs. First, piRNAs are considerably larger than most miRNAs or siRNAs, the latter two ranging from 20-23 nts in length, the former 24-30 nts. Secondly, piRNAs are produced from single stranded precursor molecules independently of Dicer processing. Like siRNAs, but not miRNAs, piRNAs are modified by DmHEN1, which results in 2'-O-methylation at the 3 ' terminus of the RNA [23,61]. Additionally, expression of piRNAs shows tissue specificity, with gonadal tissue being highly enriched for this species and associated proteins. Endogenously transcribed and processed piRNAs have been shown to be important repressors of transposable elements (TEs) in these tissues. However, expression of piRNAs has been detected in somatic tissue as well $[62,63]$.

Biogenesis of piRNAs is proposed to occur through two pathways: the primary pathway and the ping-pong dependent amplification loop. In the primary pathway, piRNAs are processed from single-stranded precursor molecules transcribed from genomic loci (piRNA clusters). In flies, primary piRNAs are associated with PIWI and Aubergine (AUB) [64], and are typically antisense to TEs [59]. Primary piRNAs produced in this manner exhibit a strong bias for a uridine residue at the 5 '-terminus of the transcript $\left(\mathrm{U}_{1}\right)$. These primary piRNAs are then fed into the ping-pong dependent amplification cycle, whereby after binding to their target transcript, cleavage occurs $10 \mathrm{nts}$ upstream from the 5 ' terminus of the primary piRNA $[65,66]$. Thus, Argonaute 3 (AGO3)-associated secondary piRNAs exhibit an adenine residue in the 10 position $\left(\mathrm{A}_{10}\right)$. Secondary piRNAs subsequently bind complementary transcripts, resulting in cleavage at the $\mathrm{A}_{10}-\mathrm{U}$ basepairing, producing piRNAs corresponding to the sequence of the initial primary piRNA they were derived from, thereby restarting the cycle.

Recently, the piRNA pathway has been implicated in antiviral immunity in invertebrates. A possible hypothesis is that the piRNA pathway can act in a compensatory manner, such as when the siRNA pathway is overburdened or suppressed. Indeed, in Drosophila, flies with a null mutant PIWI protein exhibited significantly higher titers of WNV in comparison to wild-type controls [33]. Silencing of AGO3 in An. gambiae has been shown to result in increased dissemination of ONNV [30], 
and dsRNA-knockdown of piRNA-pathway component proteins has been shown to result in increased titers of SFV in Ae. aegypti-derived Aag2 cells, with knockdown of PIWI-4 in particular showing considerable effect in this regard [67]. Additionally, sequencing of DENV2-infected Ae. albopictus C6/36 cells, which, as previously mentioned, have a dysfunctional siRNA pathway [34,35], revealed a shift from the stereotypically predominant distribution of siRNAs (19-23 nts, no significant strand bias) to products consistent with the piRNA pathway (24-30 nts, predominately positive strand, $\mathrm{A}_{10}$ bias) [68]. Wu et al. described a population of virus-derived small RNAs in their sequencing of persistently-infected Drosophila ovary somatic sheet (OSS) cells that held hallmarks of piRNAs; specifically, being between 24-30 nts in length, exhibiting a strong (95\%) strand bias, and a preference for a 5' uracil (though, notably, no bias for an $\mathrm{A}_{10}$ was seen) [69]. Similarly, 24-30 nt small RNAs produced in response to DENV2 in Ae. aegypti mosquitoes have been found by deep-sequencing [70]. Interestingly, piRNA-like small RNAs sequenced in the latter study exhibited no preference for a $5^{\prime}$ uracil, and only a slight bias for an $\mathrm{A}_{10}$ residue.

Recently, piRNA-like small RNAs exhibiting characteristics of ping-pong dependent amplification have been sequenced in mosquitoes and mosquito cells after virus infection. Morazzani et al. found that piRNA-like small RNAs are produced in the soma (non-ovarial tissue) of both Ae. aegypti and Ae. albopictus after infection with CHIKV, and that, unlike endogenously transcribed piRNAs, dsRNA was likely the biogenic precursor for virus-derived piRNAs [35]. Likewise, small RNAs from Aag2 and Ae. albopictus-derived U4.4 cells, infected with SINV, shared these same characteristics [71]. In the same study, sequencing data from C6/36 cells infected with La Crosse virus (LACV, Bunyaviridae) revealed ping-pong dependent signatures in the piRNA-like viral small RNA population. Similar results from small RNA deep-sequencing of virus infected invertebrate hosts has also been seen in Rift Valley fever virus (RVFV, Bunyaviridae) infected mosquito cells [72], as well as Schmallenberg virus (SBV, Bunyaviridae)-infected Culicoides sonorensis-derived KC cells and Aag2 cells, and blue-tongue virus (BTV, Reoviridae)-infected Culicoides and mosquito cells [73] (though notably, 24-30 nt small RNAs sequenced from BTV-infected cells did not exhibit signatures of ping-pong dependent amplification). The disparity between different virus/host pairings producing piRNAs either possessing or lacking signatures of ping-pong dependent amplification suggests that the piRNA response may be differentially modulated in response to infection by diverse viruses. While no studies profiling the piRNA response in a Culex mosquito/WNV pairing have been published, the existing data in these numerous other infection models suggest that it too may play a significant role in this system.

\subsection{WNV SfRNA as a Viral Suppressor of RNAi (VSR)}

Most arboviruses cause persistent infections within their arthropod vectors. This has led to speculation as to how these viruses maintain infection in the face of a robust RNAi response. In addition to the evolutionary mechanisms (i.e., the viral replicase error rate) described above, many plant and insect-specific viruses have developed molecular mechanisms for subverting the host RNAi response. For example, the previously mentioned FHV encodes a protein, B2, which binds to dsRNA and inhibits the function of DCR2, effectively rendering the siRNA pathway inert [74]. Plasmid-expressed LACV NSs protein has been shown to inhibit IFN and RNAi in mammalian cells and mice $[75,76]$. However, NSs fails to show any RNAi-suppressive effect in LACV-infected C6/36 
cells or in NSs plasmid-transfected U4.4 cells infected with SFV [75]. A recent publication demonstrated that DENV NS4b functions as a VSR in human Huh7 cells via inhibition of dsRNA processing by Dicer [77]. However, whether NS4b behaves similarly in mosquitoes has not been investigated, and to date, no VSR activity has been described for an arbovirus protein during mosquito infection. Thus, it is currently not clear how arboviruses establish and maintain persistent infection of arthropods.

Recently, intriguing evidence has been presented that viral subgenomic RNAs may function as VSRs in some cases. All flaviviruses studied thus far produce a sub-genomic RNA product (sfRNA) from the 3' UTR of the virus genome [78]. In WNV, this RNA comprises the last $525 \mathrm{nts}$ of the virus genome. sfRNA is produced by incomplete degradation of the viral genome by the cellular 5'-to-3' exoribonuclease XRN1, which stalls on the conserved pseudoknot-like structures present at the 5'-terminus of the 3' UTR, resulting in large amounts of sfRNA accumulating within infected cells [79,80]. Recent evidence using fluorescent reporter assays suggests that the WNV sfRNA may act as a VSR by interfering with DCR2 in a concentration-dependent manner [81], though this type of assay alone may be prone to misinterpretation, and should be corroborated by genetic rescue experiments [82]. The mechanism through which sfRNA exerts this effect is unclear, but it is hypothesized that it acts as a decoy substrate for DCR2. Interestingly, sfRNA has also been shown to act in a negative feedback loop by suppressing XRN1 activity due to the enzyme stalling at the 3' UTR [83], illustrating that multiple antiviral pathways can be manipulated by this decay product.

\section{Immune Signaling Cascades}

In addition to RNAi, there are numerous other innate immune pathways responsible for protecting insects from pathogenic organisms. These include the Toll, Immune Deficiency (Imd) and the Janus kinase (Jak)/signal transducer and activator of transcription (STAT) pathways, as well as the phenoloxidase (PO) cascade. Early characterization of these pathways revealed that the Toll pathway was activated upon challenge with gram-positive bacteria and fungi whereas the Imd pathway is responsive to gram-negative bacteria. In each case, signal transduction events initiated upon recognition of PAMPs by pattern recognition receptors (PRRs) results in the transcription of downstream effector molecules, specifically antimicrobial peptides (AMPs). The specific factors responsible for Jak/STAT activation and the downstream effector molecules are not well characterized, but numerous gene products are transcriptionally controlled by this pathway. The PO cascade is integral in wound healing and melanization of pathogenic organisms. Activation of PO results from cuticular damage or PAMP recognition. Traditionally thought to confer protection against bacterial, fungal and parasitic pathogens, recent evidence suggests that these pathways may also play a role in antiviral immunity. The details of these pathways have been extensively reviewed previously [84,85].

\subsection{Toll Pathway}

The Toll pathway was originally described in Drosophila as an evolutionarily conserved signaling cascade involved in the establishment of the dorso-ventral axis as well as in many other developmental processes $[85,86]$. It has since been characterized as having a significant role in innate immunity to gram-positive bacteria and fungi $[87,88]$. More recently, some studies suggest that the Toll pathway may serve an important role in antiviral immunity. In 2005, Zambon et al. observed a significant 
increase in the expression of the Toll regulated AMPs, Drosomycin and Metchnikowin, upon Drosophila X virus (DXV; Birnaviridae) challenge of Drosophila [89]. It was further shown that flies lacking a functional Toll pathway were significantly more susceptible to DXV challenge. Similarly, it was determined that Toll pathway components and AMPs were significantly up-regulated upon DENV infection of Ae. aegypti mosquitoes [90]. Further, suppression of Cactus (a negative regulator of Toll signaling) or MyD88 (a Toll signaling adapter protein) resulted in modest but significant reductions and accumulations of infectious DENV particles in the mosquito midguts, respectively [91]. These findings were confirmed by another study in which Toll pathway components were up-regulated upon DENV infection of Ae. aegypti salivary glands [92]. In addition, a modest increase in Dif (a Toll inducible $\mathrm{NF}-\mathrm{\kappa B}$ transcription factor) was recognized during early stages of SINV infection of Ae. aegypti [93]. Together, these data suggest that the Toll pathway plays a role in antiviral immunity in insects; however, others have observed conflicting results [94-96].

Colpitts et al. observed significant reductions in the expression levels of the Ae. aegypti ortholog of Drosophila Spätzle 5 (an upstream signaling peptide of the Toll pathway) upon Ae. aegypti infection with three flaviviruses; WNV, yellow fever virus (YFV, Flaviviridae) and DENV [94]. In addition, transcript levels of the Ae. aegypti ortholog to Drosophila Toll was reduced upon YFV infection. These findings challenge those previously published by Xi et al. [90]. Many confounding factors may account for this difference, including but not limited to experimental design, mosquito strains, virus strains and/ or environmental conditions. The authors of this study did not directly address these discrepancies, but rather suggested that viral-associated reductions potentially indicate an evolved mechanism by which arboviruses suppress antiviral pathways. However, there is currently no evidence supporting this. Using the SFV-Aedes U4.4 cell system, Fragkoudis et al. observed a down-regulation of Toll pathway component transcript levels upon infection by SFV. Furthermore, prior activation of the Toll pathway did not seem to adversely affect SFV replication in U4.4 cells [95]. From these studies it is difficult to determine with confidence the contribution of the Toll pathway to antiviral immunity of insects as a whole. However, these findings may indicate that Toll-mediated antiviral activity is specific to each virus-insect pairing. With regards to WNV, little is known about the role of this pathway during infection of insects except for the apparent down-regulation of the Spätzle5-like cytokine during infection of Ae. aegypti mosquitoes and that none of the canonical Toll pathway genes and/or associated AMPs were significantly altered during WNV infection of Cx. quinquefasciatus [96]. Further research will be needed to fully elucidate the possible contributions of Toll pathway mediated antiviral immunity during WNV infection of mosquitoes.

\subsection{Immune Deficiency (Imd) Pathway}

The Imd pathway is another immune signaling cascade of insects and bears striking similarities to the Tumor Necrosis Factor (TNF) pathway of mammals. It was initially described after the identification of a mutant Drosophila line that had significantly decreased levels of numerous AMPs, yet maintained normal levels of another AMP, Drosomycin [87,97,98]. These findings indicated that the expression of AMPs was controlled by two or more regulatory cascades. It was later determined that imd mutant flies were highly susceptible to infection with gram-negative bacteria yet maintain resistance to gram-positive bacteria and fungi [85]. Recently, the Imd pathway has been implicated in antiviral immunity in insects. The first indication of the potential significance of the Imd pathway 
during viral infections was observed in the Drosophila, cricket paralysis virus (CrPV; Dicistroviridae) model [99]. Somewhat paradoxically, the authors found that while AMPs were not up-regulated during infection with CrPV, suggesting that neither the Imd or Toll pathways were responsive to CrPV infection, mutant flies lacking components of the Imd pathway were more susceptible to viral infection resulting in shortened lifespan and increased viral replication. Together, these data suggest that Imd activation is uncoupled from AMP induction during infection with $\mathrm{CrPV}$, and implies that induction of the Imd pathway results in transcription of several other genes with presently unknown roles in immunity [100]. The role of the Imd pathway in antiviral immunity is further supported by the observations of Avadhanula et al. and Huang et al. Using a novel SINV replicon transgenic Drosophila line, the authors observed increased AMP expression as well as a modest but significant increase in susceptibility of Imd-deficient lines to SINV challenge [101,102]. Additionally, Sigma virus (SIGMAV; Rhabdoviridae) infection of Drosophila induced the expression of numerous Imd controlled AMPs; however, the significance of these observations on SIGMAV replicative fitness or fly survivorship were not assessed [103]. While there have been several studies implicating the role of Imd during antiviral immunity, there have been just as many suggesting otherwise. The induction of almost all AMPs were observed during DXV infection of Drosophila, yet mutant flies lacking a functional Rel (an Imd inducible transcription factor) were no more susceptible to DXV challenge than the controls [89]. Further, a lack of AMP induction was observed during DCV infection of Drosophila [53,104]. As with the Toll pathway, it is difficult to discern the significance of the Imd pathway in antiviral immunity based on these studies. This uncertainty is further confounded by the fact that the majority of these viruses do not naturally infect Drosophila, with the exception of SIGMAV and DCV.

These ambiguities in the literature also encompass mosquito-virus interactions. Recently, a cecropin-like peptide presumed to be under the control of the Imd signaling cascade was found to be significantly induced upon DENV infection of Ae. aegypti salivary glands [92]. Characterization of this peptide revealed that it could potently inhibit DENV and CHIKV replication in mosquito cell culture. However, the significance of its antiviral effect has not been confirmed with in vivo knock-down studies. In addition, other studies have observed either down-regulation or insignificant differences in the expression of Imd-controlled AMPs or pathway components during SFV infection of Aedes U4.4 cells or ONNV infection of An. gambiae [95,105], although in the former study, decreased SFV replication in Imd and Jak/STAT activated U4.4 cells was observed. The biological relevance of these findings is difficult to determine at this time because functional assays assessing their significance during natural infections of adult mosquitoes have not been performed. Studies investigating the role of Imd during WNV infection of mosquitoes are lacking, and it remains to be seen what if any effect this pathway has on shaping antiviral immunity to WNV in relevant mosquito vectors; however, it was demonstrated that Imd gene transcripts and/or associated AMP transcripts were unaltered during WNV infection of Cx. quinquefasciatus [96].

\subsection{Jak/STAT Pathway}

The Jak/STAT pathway is an evolutionarily conserved pathway first described for its role in embryonic segmentation in Drosophila [106]. Subsequently it was determined that it has an important role in antibacterial defense. It is comprised of the three major components, the receptor Domeless, the 
Janus Kinase (Jak) Hopscotch, and the transcription factor STAT [106-108]. Unlike the Toll and Imd pathways, a well characterized subset of inducible effector AMPs have not been associated with this pathway; however, a handful of inducible genes containing a STAT binding site in their promoters have been identified, with some of these gene products appearing to have antiviral effects.

The initial findings implicating a role of Jak/STAT in antiviral immunity in insects were observed in the Drosophila - DCV model. By performing microarray analysis on bacterial, fungal, and DCV challenged flies, the authors identified a subset of gene products that were up-regulated during DCV infection but not during fungal or bacterial challenge [104]. Upon closer inspection the authors identified a gene, vir-1, that contained the STAT binding site within its promoter and was strongly induced during DCV and FHV challenge. Furthermore, it was demonstrated that Jak/STAT deficient flies were more susceptible to DCV challenge as determined by increased DCV replication and increased mortality. These results were confirmed by studies performed by Kemp et al. who also demonstrated that the Jak/STAT pathway was important in Drosophila antiviral immunity to CrPV [109]. Interestingly, when Jak/STAT deficient flies were challenged with five other evolutionarily divergent viruses, including SINV, DXV and FHV, the authors found no effect on the survivorship of the flies. These results indicate that Jak/STAT involvement in antiviral immunity may be specific to each virus-insect pairing and not broadly applicable to all systems.

A role for Jak/STAT involvement in mosquito immunity to arboviruses has been described. Specifically, components of the Jak/STAT pathway as well as Jak/STAT inducible gene products were found to be up-regulated upon DENV infection of Ae. aegypti mosquitoes [90,110]. Included in these findings were two novel Jak/STAT inducible genes termed Dengue Virus Restriction Factors 1 and 2 (DVRF1-2). Further, it was demonstrated that suppression of these two genes resulted in 2.5- and 2.2-fold increases in DENV-2 replication in mosquitoes, respectively [110]. In addition, a recent study demonstrated that WNV induces the expression of vago in Hsu cells. Subsequent silencing of vago increased WNV titers. The antiviral effect of vago expression was determined to arise from downstream vir-1 activation via vago-induced Jak/STAT signaling. Studies in mosquitoes will be needed to validate the in vivo role of vago in controlling arbovirus infection. As with the Toll and Imd pathways others have observed conflicting data on the role of Jak/STAT pathway in antiviral immunity. In these studies there were no indications of Jak/STAT up-regulation upon infection in four separate virus-vector models (ONNV/An. gambiae, SFV/Aedes U4.4 cells, WNV-DENV-YFV/Ae. aegypti, WNV/CX. quinquefasciatus) [94-96,105]. However, as previously mentioned, activation of the Jak/STAT pathway prior to SFV infection resulted in reduced viral replication in U4.4 cells. From these studies it is difficult to accurately assess the importance of the Jak/STAT pathway in antiviral immunity. Future studies assessing the importance of this pathway on arboviral infection, dissemination and transmission rates within mosquitoes will help clarify its significance. In the context of WNV and mosquitoes, this holds true especially considering the systems utilized; however, it is difficult to determine the relevance considering that one study was limited to cell culture and the other in an ancillary vector. Additional research into the role of Jak/STAT in antiviral immunity in primary mosquito vectors is clearly required in order to fully understand its possible influence on mosquito antiviral responses. 


\subsection{Phenoloxidase (PO) Cascade}

Among arthropods, the PO cascade is an evolutionarily conserved extracellular pathway responsible for wound healing and melanization of bacterial and parasitic pathogens [84]. This pathway can be induced by cuticular damage or upon recognition of PAMPs. This in turn activates a serine protease cascade ultimately resulting in the activation of the prophenoloxidase activating enzyme (PPAE). Active PPAE cleaves the prophenoloxidase zymogen to produce PO, which catalyzes the conversion of mono- and diphenolic substrates to quinones, which is then converted to melanin [84]. While direct melanization of viruses has not been observed, there is evidence that by-products of the pathway may have antiviral effects. It was demonstrated that plasma from the tobacco budworm has virucidal effects on Helicoverpa zea single capsid nucleopolyhedrovirus (HzSNPV; Baculoviridae) and chemical inhibition of PO resulted in increased viral titers [111,112]. It was also determined that 5,6-dihydroxyindole, a byproduct of the PO cascade, could almost completely inactivate Autographa californica nucleopolyhedrovirus (AcMNPV; Baculoviridae) in vitro [113]. Similar antiviral activity has been observed in mosquitoes. Tamang et al. observed increased SINV titers in Armigeres subalbatus mosquitoes after suppression of prophenoloxidase I [114]. Such observations were also found during SFV infection of U4.4 cells. Specifically, recombinant SFV over-expressing an inhibitor of the PO cascade was able to replicate to significantly higher titers than the control virus. It was further determined that inhibition of the PO cascade could decrease the survivorship of SFV infected Ae. aegypti [115]. The authors went on to demonstrate that the specific effector molecules involved in the antiviral effects were the pathway intermediate quinones. The specific nature of their antiviral effect remains to be determined. Together, these studies highlight the potential role of the PO cascade in insect immunity to arboviruses. These are the first studies to assess the role of the PO cascade in antiviral immunity in mosquitoes and it will be interesting to see if this antiviral effect is conserved among other virus-vector pairings. Future research into the involvement PO cascade during WNV infection of Culex spp. mosquitoes is warranted.

\section{Cellular Processes}

In addition to RNAi and the PAMP-induced signaling cascades previously discussed, evidence suggests that multi-functional cellular processes can have significant effects on arboviruses in arthropods. Cellular processes, such as apoptosis and autophagy, are important in maintaining homeostasis in multicellular organisms and integral to their development. Autophagy, in effect, is the recycling center of the cell. It serves an important role in removing damaged organelles and protein aggregates which can put undue stress on the cell, ultimately reducing their constituent macromolecules to basic molecular building blocks and sources of energy. In addition, it appears to function as an innate immune defense against numerous prokaryotic and eukaryotic intracellular pathogens [116,117]. Interestingly, the role of autophagy during viral infections is not well defined. In some systems, autophagy functions in an antiviral capacity while in others it can be commandeered and utilized in a pro-viral manner [118]. However, to date, little is known about the role of autophagy during arboviral infections of arthropods with the exception of the studies discussed below, which indicate a role in antiviral immunity. Apoptosis is the process of programmed cell death (PCD) which plays an integral role in eliminating old, injured or defective cells from organisms. This process can be 
differentiated from necrosis by its ability to control the release of cellular components in apoptotic bodies which can be scavenged by phagocytic cells and thereby diminishing any potential immunologic over-reaction [119]. It has been observed that such an approach to stress-induced cell death could predispose viruses and their associated PAMPs to antigen presenting cells. Not coincidently many viruses have devised mechanisms by which to subvert or avoid apoptosis altogether [120]. In recent years it has become evident that apoptosis may function as an antiviral defense in insects especially with regards to arboviruses.

\subsection{Autophagy}

Autophagy is an evolutionarily conserved pathway that serves an important role in maintaining cellular homeostasis and cell survival [121]. Induction of autophagy results in the formation of double-phospholipid membrane vesicles termed autophagosomes which sequester the targeted organelles and proteins. Subsequently, the autophagosomes fuse with lysosomes forming autolysosomes which mediate degradation of the contents [122]. During normal growth conditions autophagy maintains cellular homeostasis by degrading unwanted or damaged organelles and protein aggregates. In times of cellular stress, autophagy catabolizes these cellular components thereby generating a pool of energy and macromolecules that maintain crucial cellular functions until favorable growth conditions return [121]. In addition, it appears to play a significant role in antiviral immunity in invertebrates. Shelly et al. demonstrated that deletion of autophagy-related genes (Atg's) increased vesicular stomatitis virus (VSV; Rhabdoviridae) replication and decreased Drosophila survivorship [123]. It was later demonstrated that pre-formed PAMPs in the context of UV-inactivated virus appear to interact with Toll-7 at the plasma membrane which leads to the activation of autophagy independently of the canonical Toll pathway [124]. These studies were the first to examine the antiviral role of autophagy to an arbovirus in the context of an invertebrate host. Additional studies are required in order to clarify whether these findings can be replicated in natural virus-vector pairings.

\subsection{Apoptosis}

Apoptosis is a conserved mechanism of programmed cell death in multicellular organisms [125] that is critical to a variety of biological processes including embryonic development, maintenance of homeostasis, and lysis of virus-infected cells by cytotoxic T-lymphocyes [119]. In vertebrates, apoptosis may be induced through two known pathways: the extrinsic, or death-receptor pathway, and the intrinsic, or mitochondrial pathway [126], though in dipterans only the intrinsic pathway is known to exist [127].

Many viruses from diverse families encode anti-apoptotic genes, such as inhibitors of apoptosis (IAPs), p53-binding proteins, and bcl-2 homologs. This has led to the hypothesis that apoptosis functions as an innate immune pathway in response to viral infection [120]. FHV strongly induces apoptotic events in Drosophila DL-1 cells through depletion of the Drosophila inhibitor-of-apoptosis-1 (DIAP1), a negative regulator of initiator caspase DRONC and effector caspase DrICE; however replication of FHV is not negatively impacted by apoptosis [128]. Similar depletion of host cell IAPs were observed in DL-1 cells [129] and Spodoptera frugiperda (order Lepidoptera) SF21 cells [129,130] infected with AcMNPV, which encodes its own viral IAP, p35, with depletion of IAPs in both cell 
lines being triggered by viral DNA replication [129]. However, p35-deficient mutant viruses demonstrated reduced infectivity of SF21 cells, and reduced infectivity and lethality in Spodoptera frugiperda larvae [131]. Furthermore, studies in flies infected with FHV or AcMNPV reveal that rapid induction of apoptosis by the upstream regulator p53 limits viral gene expression and proliferation [132], further highlighting the importance of apoptosis in controlling virus infection in these systems.

Numerous studies have investigated the role apoptosis plays in arbovirus infection models. Apoptosis is a natural consequence of arbovirus infection in mammalian cells, where cytopathic effect (CPE) is frequently seen; conversely, most arboviruses are thought to cause minimal cytopathology in insect cells, instead resulting in non-lytic, persistent infections [133,134]. However, cell death consistent with apoptosis has been observed in mosquito midgut and salivary gland tissues following infection with a variety of arboviruses [135-142], including WNV. With regards to WNV, apoptosis in the midgut epithelium [138] or salivary glands [136,142] of Cx. pipiens or Cx. quinquefasciatus mosquitoes was associated with a resistance to infection or reduced ability to transmit virus, respectively. Thus, an attractive hypothesis is that apoptosis functions in an antiviral capacity to arbovirus infection in mosquitoes, and that it may be more likely to occur in mosquitoes incapable of vectoring a particular virus rather than permissive hosts $[143,144]$. Indeed, pro-apoptotic genes in a refractory strain of Ae. aegypti mosquitoes were significantly up-regulated between 24 and $48 \mathrm{~h}$ postinfection (hpi) in response to DENV2 infection as compared to a susceptible strain of Ae. aegypti or blood-only fed mosquitoes from the same strain [145]. In the same study, dsRNA-knockdown of the caspase inhibitor AeIAP1 in two susceptible strains converted the phenotype from susceptible to refractory, and knockdown of initiator-caspase AeDronc in the refractory strain resulted in increased permissiveness to DENV2 infection. The same group previously reported up-regulation of pro-apoptotic genes in the DENV2-susceptible strain in comparison to the refractory strain at $48 \mathrm{hpi}$ [146], indicating that apoptotic events controlling virus infection may be induced acutely and rapidly limit the course of infection. However, it is important to note that neither of these studies directly measured apoptotic events in midgut tissue after DENV2 infection, and that the methods used to measure differential gene expression were different between studies (qPCR and suppressive subtractive hybridization, respectively [145,146]). Conversely, modulation of apoptosis by RNAi-mediated silencing of either apoptosis inhibitors or initiators in Ae. Aegypti, subsequently infected with SINV, produced results contrary to the hypothesis that apoptosis acts in an antiviral manner to arbovirus infection in mosquitoes [147]. Specifically, inhibition of apoptosis led to decreased infection and dissemination rates, and induction of apoptosis led to greater infection and dissemination. The authors speculate that experimental systemic induction or inhibition of apoptosis prior to virus infection, in contrast to apoptosis being stimulated in individual cells in response to virus infection, resulting in widespread destruction of structural barriers to viral infection/dissemination, may account for this apparent discrepancy. In summary, the variety of studies in mosquitoes investigating the role of apoptosis in response to arbovirus infection strongly implicate the pathway as contributing to the acute antiviral response; and that either the presence or lack of an effective apoptotic response has important consequences for vector competence in mosquitoes. 


\section{Conclusions}

To date, little work has been done elucidating the contributions of each of the aforementioned pathways with regards to WNV infection of mosquitoes. However, based on the evolutionary relatedness of the virus/invertebrate systems analyzed, informed conclusions can be made with regards to the mosquito's innate immune response to WNV. Specifically, it is evident from these studies that the exo-siRNA pathway most likely contributes significantly to suppression of WNV replication in mosquitoes and their survivorship. Numerous studies in Drosophila and mosquitoes have demonstrated the importance of the exo-siRNA response in innate antiviral immunity in invertebrates [27-29] and work with Culex mosquitoes indicates that the exo-siRNA pathway actively targets the WNV genome [36], but the influence of this pathway on vector competence remains unclear. The extent to which other RNAi pathways, specifically the piRNA and systemic RNAi pathways, contribute to mosquito innate anti-WNV immunity and vector competence remains to be determined. The findings from such studies will be integral to fully elucidating the contributions of RNAi in mosquito antiviral immunity.

The involvement of other immune pathways, specifically the immune signaling cascades (e.g., Jak/STAT, Imd, Toll, PO) and cellular processes (e.g., autophagy and apoptosis), in mosquito anti-WNV immunity and vector competence is difficult to quantify at this time. While some evidence suggests that the Jak/STAT and Imd pathways and apoptosis may function to limit WNV infection of mosquitoes, their relevance is somewhat diminished because the studies were limited to either cell culture systems or are predicated on observational correlations. Furthermore, the evidence implicating their involvement is highly inconsistent. For instance, while there were no indications of Jak/STAT induction during WNV infection of Ae. aegypti in one study, others observed anti-WNV activity associated with the induction of Jak/STAT by vago during WNV infection of Hsu cells [54,94]. It has been demonstrated that these observed inconsistencies may result from the inherent variability between virus-invertebrate pairings [109]. Evidence supporting the role of these non-RNAi based immune pathways in antiviral immunity has been described in other arbovirus-mosquito pairings. However, in addition to the inter-study inconsistencies, many of these studies observed that most of these pathways exert marginal antiviral activity. For example, the silencing of components of the Jak/STAT pathway in Ae. Aegypti, resulted in an approximately half a log increase in DENV titers [110]. The significance of these findings is not in doubt; however, the question remains: Are these non-RNAi-based immune pathways biologically relevant in invertebrate antiviral immunity and/or vector competence? We would assume that if these pathways were biologically relevant that we would be able to identify evolved signatures of antiviral immune recognition and downstream effector molecules. It is conventionally thought that some if not all of these pathways are induced upon recognition of PAMPs by PRRs. For both RNAi and viral induced autophagy this paradigm has been validated in invertebrates [27,124]; however, no known viral-specific PRRs and/or viral-derived PAMPs have been identified for the other pathways. It could be that such PRRs and/or PAMPs exist, but have yet to be characterized. In addition, only a couple of downstream antiviral effector molecules have been described and their mechanisms of action have yet to be determined [92,104,110]. It will be important that future studies focus on elucidating the signatures of viral immune recognition and the mode of action of downstream effector molecules in order to fully appreciate their involvement in antiviral immunity. 
The possibility remains that such PRR/PAMP interactions are not involved in the induction of these pathways during viral infection of invertebrates. There is an increasing body of literature suggesting that other global stress factors may be controlling the induction of these pathways. Viruses and hosts are locked in an evolutionary arms race with hosts having evolved numerous innate sensing mechanisms and viruses having evolved countermeasures to either diminish their functionality (e.g., VSRs) or ways of evading them all together (e.g., genome sequestration or dsRNA degradation) $[74,148,149]$. While these countermeasures can be very effective, often times they are very specialized and are unable to conceal broader insults associated with the lifecycle of viruses (e.g., membrane perturbations associated with viral entry). In mammalian cells, several studies have demonstrated that such insults can lead to the induction of interferon stimulated genes independent of interferon production [150-152]. Further, perturbations in cytoskeletal integrity and functionality have been shown to induce innate immune responses in mammalian cells $[153,154]$. During viral infections, the cytoskeleton functions not only as physical barrier, but can be important during viral entry and in transporting viral components throughout the cell [155]. This viral-induced stress could inadvertently induce a global, non-descript innate immune response [156]. In addition, many viruses induce the production of reactive oxygen species (ROS) which have been linked to innate immune signaling pathways [157-159]. In fact, oxidative stress has been observed in C6/36 Ae. albopictus cells during DENV infection and in Drosophila during SINV and FHV infections [109,160]. In addition, Pan et al. demonstrated that the Toll pathway can be activated in Ae. aegypti upon ROS induction during a Wolbachia infection, illustrating the link between the induction of global stress signals and downstream immune signaling cascades [161]. Interestingly, it was observed that Wolbachia infections can reduce WNV replication in Cx. quinquefasciatus [162]. Further, viruses have been shown to impart endoplasmic reticulum (ER) stress on infected cells which has been associated with antiviral signaling. ER-stress is a broad term that encompasses multiple stress response pathways, all of which converge upon the unfolded protein response (UPR) which subsequently result in immune activation and can often times result in apoptosis $[163,164]$. Interestingly, it appears that the UPR is up-regulated during DENV infection of mosquito cells and numerous studies have evaluated this response to arboviruses in mammalian cells [165-169]. Currently, it is unknown whether or not these pathways, individually or synergistically, contribute to and account for the discrepancies concerning the antiviral nature of many of these pathways. Therefore, it will be important that future studies examining these pathways consider the possibility that non-canonical activation of immune pathways and/or non-specific global stressors could be responsible for the induction and/or activity of these pathways.

We will conclude by reiterating the statement we made in the introductory paragraph: while a continually expanding body of knowledge is developing regarding mosquito innate immunity to arbovirus infection, there are still major gaps in our knowledge regarding the relevance of these pathways to virus-vector ecology and natural transmission dynamics. While well-established models such as Drosophila have been greatly useful due to being easily amenable to genetic manipulation, it is important that relevant virus-vector pairings be investigated to draw sound conclusions that can be extrapolated to the natural world. This effort will be aided with the recent completion of the genome sequences for the three most medically relevant mosquito species, Aedes aegypti, Anopheles gambiae, and Culex quinquefasciatus [170-172]. 


\section{Acknowledgements}

The authors are funded by National Institutes of Health (NIH) award AI67380. In addition, the authors would like to thank the members of the Ebel lab for helpful discussions regarding the content of this review.

\section{Conflicts of Interest}

The authors declare no conflict of interest.

\section{References and Notes}

1. Kramer, L.D.; Ebel, G.D. Dynamics of flavivirus infection in mosquitoes. Adv. Virus Res. 2003, 60, 187-232.

2. Kilpatrick, A.M.; Randolph, S.E. Drivers, dynamics, and control of emerging vector-borne zoonotic diseases. Lancet 2012, 380, 1946-1955.

3. Kilpatrick, A.M.; Fonseca, D.M.; Ebel, G.D.; Reddy, M.R.; Kramer, L.D. Spatial and temporal variation in vector competence of Culex pipiens and $C x$. restuans mosquitoes for West Nile virus. Am. J. Trop. Med. Hyg. 2010, 83, 607-613.

4. Kilpatrick, A.M.; Meola, M.A.; Moudy, R.M.; Kramer, L.D. Temperature, viral genetics, and the transmission of West Nile virus by Culex pipiens mosquitoes. PLoS Pathog. 2008, 4, e1000092.

5. Samuel, M.A.; Diamond, M.S. Alpha/beta interferon protects against lethal West Nile virus infection by restricting cellular tropism and enhancing neuronal survival. J. Virol. 2005, 79, 13350-13361.

6. Daffis, S.; Samuel, M.A.; Suthar, M.S.; Gale, M., Jr.; Diamond, M.S. Toll-like receptor 3 has a protective role against West Nile virus infection. J. Virol. 2008, 82, 10349-10358.

7. Ben-Nathan, D.; Huitinga, I.; Lustig, S.; van Rooijen, N.; Kobiler, D. West Nile virus neuroinvasion and encephalitis induced by macrophage depletion in mice. Arch. Virol. 1996, 141, 459-469.

8. Durrant, D.M.; Robinette, M.L.; Klein, R.S. IL-1R1 is required for dendritic cell-mediated T cell reactivation within the CNS during West Nile virus encephalitis. J. Exp. Med. 2013, 210, 503-516.

9. Ma, D.Y.; Suthar, M.S.; Kasahara, S.; Gale, M., Jr.; Clark, E.A. CD22 is required for protection against West Nile virus Infection. J. Virol. 2013, 87, 3361-3375.

10. Diamond, M.S.; Shrestha, B.; Marri, A.; Mahan, D.; Engle, M. B cells and antibody play critical roles in the immediate defense of disseminated infection by West Nile encephalitis virus. J. Virol. 2003, 77, 2578-2586.

11. Mehlhop, E.; Diamond, M.S. Protective immune responses against West Nile virus are primed by distinct complement activation pathways. J. Exp. Med. 2006, 203, 1371-1381.

12. Fuchs, A.; Pinto, A.K.; Schwaeble, W.J.; Diamond, M.S. The lectin pathway of complement activation contributes to protection from West Nile virus infection. Virology 2011, 412, 101-109.

13. Mehlhop, E.; Fuchs, A.; Engle, M.; Diamond, M.S. Complement modulates pathogenesis and antibody-dependent neutralization of West Nile virus infection through a C5-independent mechanism. Virology 2009, 393, 11-15. 
14. Bosio, C.F.; Fulton, R.E.; Salasek, M.L.; Beaty, B.J.; Black, W.C.T. Quantitative trait loci that control vector competence for dengue-2 virus in the mosquito Aedes aegypti. Genetics 2000, 156, $687-698$.

15. Bennett, K.E.; Flick, D.; Fleming, K.H.; Jochim, R.; Beaty, B.J.; Black, W.C.T. Quantitative trait loci that control dengue-2 virus dissemination in the mosquito Aedes aegypti. Genetics 2005, 170, 185-194.

16. Richards, S.L.; Anderson, S.L.; Lord, C.C.; Smartt, C.T.; Tabachnick, W.J. Relationships between infection, dissemination, and transmission of West Nile virus RNA in Culex pipiens quinquefasciatus (Diptera: Culicidae). J. Med. Entomol. 2012, 49, 132-142.

17. Lindbo, J.A.; Silva-Rosales, L.; Proebsting, W.M.; Dougherty, W.G. Induction of a highly specific antiviral state in transgenic plants: Implications for regulation of gene expression and virus resistance. Plant Cell 1993, 5, 1749-1759.

18. Ratcliff, F.; Harrison, B.D.; Baulcombe, D.C. A similarity between viral defense and gene silencing in plants. Science 1997, 276, 1558-1560.

19. Fire, A.; Xu, S.; Montgomery, M.K.; Kostas, S.A.; Driver, S.E.; Mello, C.C. Potent and specific genetic interference by double-stranded RNA in Caenorhabditis elegans. Nature 1998, 391, 806-811.

20. Kennerdell, J.R.; Carthew, R.W. Use of dsRNA-mediated genetic interference to demonstrate that frizzled and frizzled 2 act in the wingless pathway. Cell 1998, 95, 1017-1026.

21. Liu, Q.; Rand, T.A.; Kalidas, S.; Du, F.; Kim, H.E.; Smith, D.P.; Wang, X. R2D2, a bridge between the initiation and effector steps of the Drosophila RNAi pathway. Science 2003, 301, 1921-1925.

22. Yang, Z.; Ebright, Y.W.; Yu, B.; Chen, X. HEN1 recognizes 21-24 nt small RNA duplexes and deposits a methyl group onto the 2' $\mathrm{OH}$ of the $3^{\prime}$ terminal nucleotide. Nucleic Acids Res. 2006, 34, 667-675.

23. Horwich, M.D.; Li, C.; Matranga, C.; Vagin, V.; Farley, G.; Wang, P.; Zamore, P.D. The Drosophila RNA methyltransferase, DmHen1, modifies germline piRNAs and single-stranded siRNAs in RISC. Curr. Biol. 2007, 17, 1265-1272.

24. Rand, T.A.; Ginalski, K.; Grishin, N.V.; Wang, X. Biochemical identification of Argonaute 2 as the sole protein required for RNA-induced silencing complex activity. Proc. Natl. Acad. Sci. USA 2004, 101, 14385-14389.

25. Pusch, O.; Boden, D.; Silbermann, R.; Lee, F.; Tucker, L.; Ramratnam, B. Nucleotide sequence homology requirements of HIV-1-specific short hairpin RNA. Nucleic Acids Res. 2003, 31, 6444-6449.

26. Westerhout, E.M.; Ooms, M.; Vink, M.; Das, A.T.; Berkhout, B. HIV-1 can escape from RNA interference by evolving an alternative structure in its RNA genome. Nucleic Acids Res. 2005, 33, 796-804.

27. Aliyari, R.; Wu, Q.; Li, H.W.; Wang, X.H.; Li, F.; Green, L.D.; Han, C.S.; Li, W.X.; Ding, S.W. Mechanism of induction and suppression of antiviral immunity directed by virus-derived small RNAs in Drosophila. Cell Host Microbe 2008, 4, 387-397.

28. Galiana-Arnoux, D.; Dostert, C.; Schneemann, A.; Hoffmann, J.A.; Imler, J.L. Essential function in vivo for Dicer-2 in host defense against RNA viruses in Drosophila. Nat. Immunol. 2006, 7 , 590-597. 
29. Van Rij, R.P.; Saleh, M.C.; Berry, B.; Foo, C.; Houk, A.; Antoniewski, C.; Andino, R. The RNA silencing endonuclease Argonaute 2 mediates specific antiviral immunity in Drosophila melanogaster. Genes Dev. 2006, 20, 2985-2995.

30. Keene, K.M.; Foy, B.D.; Sanchez-Vargas, I.; Beaty, B.J.; Blair, C.D.; Olson, K.E. RNA interference acts as a natural antiviral response to O'nyong-nyong virus (Alphavirus; Togaviridae) infection of Anopheles gambiae. Proc. Natl. Acad. Sci. USA 2004, 101, 17240-17245.

31. Myles, K.M.; Wiley, M.R.; Morazzani, E.M.; Adelman, Z.N. Alphavirus-derived small RNAs modulate pathogenesis in disease vector mosquitoes. Proc. Natl. Acad. Sci. USA 2008, 105, 19938-19943.

32. Sanchez-Vargas, I.; Scott, J.C.; Poole-Smith, B.K.; Franz, A.W.; Barbosa-Solomieu, V.; Wilusz, J.; Olson, K.E.; Blair, C.D. Dengue virus type 2 infections of Aedes aegypti are modulated by the mosquito's RNA interference pathway. PLoS Pathog. 2009, 5, e1000299.

33. Chotkowski, H.L.; Ciota, A.T.; Jia, Y.; Puig-Basagoiti, F.; Kramer, L.D.; Shi, P.Y.; Glaser, R.L. West Nile virus infection of Drosophila melanogaster induces a protective RNAi response. Virology 2008, 377, 197-206.

34. Brackney, D.E.; Scott, J.C.; Sagawa, F.; Woodward, J.E.; Miller, N.A.; Schilkey, F.D.; Mudge, J.; Wilusz, J.; Olson, K.E.; Blair, C.D.; et al. C6/36 Aedes albopictus cells have a dysfunctional antiviral RNA interference response. PLoS Negl. Trop. Dis. 2010, 4, e856.

35. Morazzani, E.M.; Wiley, M.R.; Murreddu, M.G.; Adelman, Z.N.; Myles, K.M. Production of virus-derived ping-pong-dependent piRNA-like small RNAs in the Mosquito Soma. PLoS Pathog. 2012, 8, e1002470.

36. Brackney, D.E.; Beane, J.E.; Ebel, G.D. RNAi targeting of West Nile virus in mosquito midguts promotes virus diversification. PLoS Pathog. 2009, 5, e1000502.

37. Konishi, M.; Wu, C.H.; Kaito, M.; Hayashi, K.; Watanabe, S.; Adachi, Y.; Wu, G.Y. siRNA-Resistance in treated HCV replicon cells is correlated with the development of specific HCV mutations. J. Viral. Hepat. 2006, 13, 756-761.

38. Martínez, F.; Lafforgue, G.; Morelli, M.J.; González-Candelas, F.; Chua, N.-H.; Daròs, J.-A.; Elena, S.F. Ultradeep sequencing analysis of population dynamics of virus escape mutants in RNAi-mediated resistant plants. Mol. Biol. Evol. 2012, 29, 3297-3307.

39. Gitlin, L.; Stone, J.K.; Andino, R. Poliovirus escape from RNA interference: Short interfering RNA-target recognition and implications for therapeutic approaches. J. Virol. 2005, 79, 1027-1035.

40. Jerzak, G.; Bernard, K.A.; Kramer, L.D.; Ebel, G.D. Genetic variation in West Nile virus from naturally infected mosquitoes and birds suggests quasispecies structure and strong purifying selection. J. Gen. Virol. 2005, 86, 2175-2183.

41. Coffey, L.L.; Beeharry, Y.; Borderia, A.V.; Blanc, H.; Vignuzzi, M. Arbovirus high fidelity variant loses fitness in mosquitoes and mice. Proc. Natl. Acad. Sci. USA 2011, 108, 16038-16043.

42. Voinnet, O. Non-cell autonomous RNA silencing. FEBS Lett. 2005, 579, 5858-5871.

43. Feinberg, E.H.; Hunter, C.P. Transport of dsRNA into cells by the transmembrane protein SID-1. Science 2003, 301, 1545-1547.

44. Winston, W.M.; Molodowitch, C.; Hunter, C.P. Systemic RNAi in C. elegans requires the putative transmembrane protein SID-1. Science 2002, 295, 2456-2459. 
45. Saleh, M.C.; van Rij, R.P.; Hekele, A.; Gillis, A.; Foley, E.; O’Farrell, P.H.; Andino, R. The endocytic pathway mediates cell entry of dsRNA to induce RNAi silencing. Nat. Cell Biol. 2006, 8, 793-802.

46. Saleh, M.C.; Tassetto, M.; van Rij, R.P.; Goic, B.; Gausson, V.; Berry, B.; Jacquier, C.; Antoniewski, C.; Andino, R. Antiviral immunity in Drosophila requires systemic RNA interference spread. Nature 2009, 458, 346-350.

47. Attarzadeh-Yazdi, G.; Fragkoudis, R.; Chi, Y.; Siu, R.W.; Ulper, L.; Barry, G.; Rodriguez-Andres, J.; Nash, A.A.; Bouloy, M.; Merits, A.; et al. Cell-to-cell spread of the RNA interference response suppresses Semliki Forest virus (SFV) infection of mosquito cell cultures and cannot be antagonized by SFV. J. Virol. 2009, 83, 5735-5748.

48. Sijen, T.; Fleenor, J.; Simmer, F.; Thijssen, K.L.; Parrish, S.; Timmons, L.; Plasterk, R.H.; Fire, A. On the role of RNA amplification in dsRNA-triggered gene silencing. Cell 2001, 107, 465-476.

49. Pak, J.; Fire, A. Distinct populations of primary and secondary effectors during RNAi in C. elegans. Science 2007, 315, 241-244.

50. Sijen, T.; Steiner, F.A.; Thijssen, K.L.; Plasterk, R.H. Secondary siRNAs result from unprimed RNA synthesis and form a distinct class. Science 2007, 315, 244-247.

51. Goic, B.; Vodovar, N.; Mondotte, J.A.; Monot, C.; Frangeul, L.; Blanc, H.; Gausson, V.; Vera-Otarola, J.; Cristofari, G.; Saleh, M.C. RNA-mediated interference and reverse transcription control the persistence of RNA viruses in the insect model Drosophila. Nat. Immunol. 2013, 14, 396-403.

52. Rechavi, O.; Minevich, G.; Hobert, O. Transgenerational inheritance of an acquired small RNA-based antiviral response in C. elegans. Cell 2011, 147, 1248-1256.

53. Deddouche, S.; Matt, N.; Budd, A.; Mueller, S.; Kemp, C.; Galiana-Arnoux, D.; Dostert, C.; Antoniewski, C.; Hoffmann, J.A.; Imler, J.L. The DExD/H-box helicase Dicer-2 mediates the induction of antiviral activity in drosophila. Nat. Immunol. 2008, 9, 1425-1432.

54. Paradkar, P.N.; Trinidad, L.; Voysey, R.; Duchemin, J.B.; Walker, P.J. Secreted Vago restricts West Nile virus infection in Culex mosquito cells by activating the Jak-STAT pathway. Proc. Natl. Acad. Sci. USA 2012, 109, 18915-18920.

55. Lin, H.; Spradling, A.C. A novel group of pumilio mutations affects the asymmetric division of germline stem cells in the Drosophila ovary. Development 1997, 124, 2463-2476.

56. Aravin, A.; Gaidatzis, D.; Pfeffer, S.; Lagos-Quintana, M.; Landgraf, P.; Iovino, N.; Morris, P.; Brownstein, M.J.; Kuramochi-Miyagawa, S.; Nakano, T.; et al. A novel class of small RNAs bind to MILI protein in mouse testes. Nature 2006, 442, 203-207.

57. Girard, A.; Sachidanandam, R.; Hannon, G.J.; Carmell, M.A. A germline-specific class of small RNAs binds mammalian Piwi proteins. Nature 2006, 442, 199-202.

58. Grivna, S.T.; Beyret, E.; Wang, Z.; Lin, H. A novel class of small RNAs in mouse spermatogenic cells. Genes Dev. 2006, 20, 1709-1714.

59. Saito, K.; Nishida, K.M.; Mori, T.; Kawamura, Y.; Miyoshi, K.; Nagami, T.; Siomi, H.; Siomi, M.C. Specific association of Piwi with rasiRNAs derived from retrotransposon and heterochromatic regions in the Drosophila genome. Genes Dev. 2006, 20, 2214-2222. 
60. Watanabe, T.; Takeda, A.; Tsukiyama, T.; Mise, K.; Okuno, T.; Sasaki, H.; Minami, N.; Imai, H. Identification and characterization of two novel classes of small RNAs in the mouse germline: Retrotransposon-derived siRNAs in oocytes and germline small RNAs in testes. Genes Dev. 2006, 20, 1732-1743.

61. Saito, K.; Sakaguchi, Y.; Suzuki, T.; Siomi, H.; Siomi, M.C. Pimet, the Drosophila homolog of HEN1, mediates 2'-O-methylation of Piwi-interacting RNAs at their 3' ends. Genes Dev. 2007, $21,1603-1608$.

62. Lee, E.J.; Banerjee, S.; Zhou, H.; Jammalamadaka, A.; Arcila, M.; Manjunath, B.S.; Kosik, K.S. Identification of piRNAs in the central nervous system. RNA 2011, 17, 1090-1099.

63. Yan, Z.; Hu, H.Y.; Jiang, X.; Maierhofer, V.; Neb, E.; He, L.; Hu, Y.; Hu, H.; Li, N.; Chen, W.; et al. Widespread expression of piRNA-like molecules in somatic tissues. Nucleic Acids Res. 2011, 39, 6596-6607.

64. Nishida, K.M.; Saito, K.; Mori, T.; Kawamura, Y.; Nagami-Okada, T.; Inagaki, S.; Siomi, H.; Siomi, M.C. Gene silencing mechanisms mediated by Aubergine piRNA complexes in Drosophila male gonad. RNA 2007, 13, 1911-1922.

65. Brennecke, J.; Aravin, A.A.; Stark, A.; Dus, M.; Kellis, M.; Sachidanandam, R.; Hannon, G.J. Discrete small RNA-generating loci as master regulators of transposon activity in Drosophila. Cell 2007, 128, 1089-1103.

66. Gunawardane, L.S.; Saito, K.; Nishida, K.M.; Miyoshi, K.; Kawamura, Y.; Nagami, T.; Siomi, H.; Siomi, M.C. A slicer-mediated mechanism for repeat-associated siRNA 5 ' end formation in Drosophila. Science 2007, 315, 1587-1590.

67. Schnettler, E.; Donald, C.L.; Human, S.; Watson, M.; Siu, R.W.; McFarlane, M.; Fazakerley, J.K.; Kohl, A.; Fragkoudis, R. Knockdown of piRNA pathway proteins results in enhanced Semliki Forest virus production in mosquito cells. J. Gen. Virol. 2013, 94, 1680-1689.

68. Scott, J.C.; Brackney, D.E.; Campbell, C.L.; Bondu-Hawkins, V.; Hjelle, B.; Ebel, G.D.; Olson, K.E.; Blair, C.D. Comparison of dengue virus type 2-specific small RNAs from RNA interference-competent and -incompetent mosquito cells. PLoS Negl. Trop. Dis. 2010, 4, e848.

69. Wu, Q.; Luo, Y.; Lu, R.; Lau, N.; Lai, E.C.; Li, W.X.; Ding, S.W. Virus discovery by deep sequencing and assembly of virus-derived small silencing RNAs. Proc. Natl. Acad. Sci. USA 2010, 107, 1606-1611.

70. Hess, A.M.; Prasad, A.N.; Ptitsyn, A.; Ebel, G.D.; Olson, K.E.; Barbacioru, C.; Monighetti, C.; Campbell, C.L. Small RNA profiling of Dengue virus-mosquito interactions implicates the PIWI RNA pathway in anti-viral defense. BMC Microbiol. 2011, 11, doi:10.1186/1471-2180-11-45.

71. Vodovar, N.; Bronkhorst, A.W.; van Cleef, K.W.; Miesen, P.; Blanc, H.; van Rij, R.P.; Saleh, M.C. Arbovirus-derived piRNAs exhibit a ping-pong signature in Mosquito cells. PLoS One 2012, 7, e30861.

72. Leger, P.; Lara, E.; Jagla, B.; Sismeiro, O.; Mansuroglu, Z.; Coppee, J.Y.; Bonnefoy, E.; Bouloy, M. Dicer-2 and piwi mediated RNA interference in Rift Valley Fever Virus infected Mosquito cells. J. Virol. 2012, 87, doi: 10.1128/JVI.02795-12.

73. Schnettler, E.; Ratinier, M.; Watson, M.; Shaw, A.E.; McFarlane, M.; Varela, M.; Elliott, R.M.; Palmarini, M.; Kohl, A. RNA interference targets arbovirus replication in Culicoides cells. J. Virol. 2012, 87, 2441-2454. 
74. Li, H.; Li, W.X.; Ding, S.W. Induction and suppression of RNA silencing by an animal virus. Science 2002, 296, 1319-1321.

75. Blakqori, G.; Delhaye, S.; Habjan, M.; Blair, C.D.; Sanchez-Vargas, I.; Olson, K.E.; Attarzadeh-Yazdi, G.; Fragkoudis, R.; Kohl, A.; Kalinke, U.; et al. La Crosse bunyavirus nonstructural protein NSs serves to suppress the type I interferon system of mammalian hosts. J. Virol. 2007, 81, 4991-4999.

76. Soldan, S.S.; Plassmeyer, M.L.; Matukonis, M.K.; Gonzalez-Scarano, F. La Crosse virus nonstructural protein NSs counteracts the effects of short interfering RNA. J. Virol. 2005, 79, 234-244.

77. Kakumani, P.K.; Ponia, S.S.; S, R.K.; Sood, V.; Chinnappan, M.; Banerjea, A.C.; Medigeshi, G.R.; Malhotra, P.; Mukherjee, S.K.; Bhatnagar, R.K. Role of RNA interference (RNAi) in dengue virus replication and identification of NS4B as an RNAi suppressor. J. Virol. 2013, 87, 8870-8883.

78. Pijlman, G.P.; Funk, A.; Kondratieva, N.; Leung, J.; Torres, S.; van der Aa, L.; Liu, W.J.; Palmenberg, A.C.; Shi, P.Y.; Hall, R.A.; et al. A highly structured, nuclease-resistant, noncoding RNA produced by flaviviruses is required for pathogenicity. Cell Host Microbe 2008, 4, 579-591.

79. Funk, A.; Truong, K.; Nagasaki, T.; Torres, S.; Floden, N.; Balmori Melian, E.; Edmonds, J.; Dong, H.; Shi, P.Y.; Khromykh, A.A. RNA structures required for production of subgenomic flavivirus RNA. J. Virol. 2010, 84, 11407-11417.

80. Silva, P.A.; Pereira, C.F.; Dalebout, T.J.; Spaan, W.J.; Bredenbeek, P.J. An RNA pseudoknot is required for production of yellow fever virus subgenomic RNA by the host nuclease XRN1. J. Virol. 2010, 84, 11395-11406.

81. Schnettler, E.; Sterken, M.G.; Leung, J.Y.; Metz, S.W.; Geertsema, C.; Goldbach, R.W.; Vlak, J.M.; Kohl, A.; Khromykh, A.A.; Pijlman, G.P. Noncoding flavivirus RNA displays RNA interference suppressor activity in insect and Mammalian cells. J. Virol. 2012, 86, 13486-13500.

82. Ding, S.W.; Voinnet, O. Antiviral immunity directed by small RNAs. Cell 2007, 130, 413-426.

83. Moon, S.L.; Anderson, J.R.; Kumagai, Y.; Wilusz, C.J.; Akira, S.; Khromykh, A.A.; Wilusz, J. A noncoding RNA produced by arthropod-borne flaviviruses inhibits the cellular exoribonuclease XRN1 and alters host mRNA stability. RNA 2012, 18, 2029-2040.

84. Christensen, B.M.; Li, J.; Chen, C.C.; Nappi, A.J. Melanization immune responses in mosquito vectors. Trends Parasitol. 2005, 21, 192-199.

85. Lemaitre, B.; Hoffmann, J. The host defense of Drosophila melanogaster. Annu. Rev. Immunol. 2007, 25, 697-743.

86. Anderson, K.V.; Bokla, L.; Nusslein-Volhard, C. Establishment of dorsal-ventral polarity in the Drosophila embryo: The induction of polarity by the Toll gene product. Cell 1985, 42, 791-798.

87. Lemaitre, B.; Meister, M.; Govind, S.; Georgel, P.; Steward, R.; Reichhart, J.M.; Hoffmann, J.A. Functional analysis and regulation of nuclear import of dorsal during the immune response in Drosophila. EMBO J. 1995, 14, 536-545.

88. Lemaitre, B.; Reichhart, J.M.; Hoffmann, J.A. Drosophila host defense: Differential induction of antimicrobial peptide genes after infection by various classes of microorganisms. Proc. Natl. Acad. Sci. USA 1997, 94, 14614-14619.

89. Zambon, R.A.; Nandakumar, M.; Vakharia, V.N.; Wu, L.P. The Toll pathway is important for an antiviral response in Drosophila. Proc. Natl. Acad. Sci. USA 2005, 102, 7257-7262. 
90. Xi, Z.; Ramirez, J.L.; Dimopoulos, G. The Aedes aegypti toll pathway controls dengue virus infection. PLoS Pathog. 2008, 4, e1000098.

91. Ramirez, J.L.; Dimopoulos, G. The Toll immune signaling pathway control conserved anti-dengue defenses across diverse Ae. aegypti strains and against multiple dengue virus serotypes. Dev. Comp. Immunol. 2010, 34, 625-629.

92. Luplertlop, N.; Surasombatpattana, P.; Patramool, S.; Dumas, E.; Wasinpiyamongkol, L.; Saune, L.; Hamel, R.; Bernard, E.; Sereno, D.; Thomas, F.; et al. Induction of a peptide with activity against a broad spectrum of pathogens in the Aedes aegypti salivary gland, following Infection with Dengue Virus. PLoS Pathog. 2011, 7, e1001252.

93. Sanders, H.R.; Foy, B.D.; Evans, A.M.; Ross, L.S.; Beaty, B.J.; Olson, K.E.; Gill, S.S. Sindbis virus induces transport processes and alters expression of innate immunity pathway genes in the midgut of the disease vector, Aedes aegypti. Insect Biochem. Mol. Biol. 2005, 35, 1293-1307.

94. Colpitts, T.M.; Cox, J.; Vanlandingham, D.L.; Feitosa, F.M.; Cheng, G.; Kurscheid, S.; Wang, P.; Krishnan, M.N.; Higgs, S.; Fikrig, E. Alterations in the Aedes aegypti transcriptome during infection with West Nile, dengue and yellow fever viruses. PLoS Pathog. 2011, 7, e1002189.

95. Fragkoudis, R.; Chi, Y.; Siu, R.W.; Barry, G.; Attarzadeh-Yazdi, G.; Merits, A.; Nash, A.A.; Fazakerley, J.K.; Kohl, A. Semliki Forest virus strongly reduces mosquito host defence signaling. Insect Mol. Biol. 2008, 17, 647-656.

96. Bartholomay, L.C.; Waterhouse, R.M.; Mayhew, G.F.; Campbell, C.L.; Michel, K.; Zou, Z.; Ramirez, J.L.; Das, S.; Alvarez, K.; Arensburger, P.; et al. Pathogenomics of Culex quinquefasciatus and meta-analysis of infection responses to diverse pathogens. Science 2010, 330, 88-90.

97. Corbo, J.C.; Levine, M. Characterization of an immunodeficiency mutant in Drosophila. Mech. Dev. 1996, 55, 211-220.

98. Levashina, E.A.; Ohresser, S.; Lemaitre, B.; Imler, J.L. Two distinct pathways can control expression of the gene encoding the Drosophila antimicrobial peptide metchnikowin. J. Mol. Biol. 1998, 278, 515-527.

99. Costa, A.; Jan, E.; Sarnow, P.; Schneider, D. The Imd pathway is involved in antiviral immune responses in Drosophila. PLoS One 2009, 4, e7436.

100. De Gregorio, E.; Spellman, P.T.; Tzou, P.; Rubin, G.M.; Lemaitre, B. The Toll and Imd pathways are the major regulators of the immune response in Drosophila. EMBO J. 2002, 21, 2568-2579.

101. Avadhanula, V.; Weasner, B.P.; Hardy, G.G.; Kumar, J.P.; Hardy, R.W. A novel system for the launch of alphavirus RNA synthesis reveals a role for the Imd pathway in arthropod antiviral response. PLoS Pathog. 2009, 5, e1000582.

102. Huang, Z.; Kingsolver, M.B.; Avadhanula, V.; Hardy, R.W. An antiviral role for antimicrobial peptides during the arthropod response to alphavirus replication. J. Virol. 2013, 87, 4272-4280.

103. Tsai, C.W.; McGraw, E.A.; Ammar, E.D.; Dietzgen, R.G.; Hogenhout, S.A. Drosophila melanogaster mounts a unique immune response to the Rhabdovirus sigma virus. Appl. Environ. Microbiol. 2008, 74, 3251-3256.

104. Dostert, C.; Jouanguy, E.; Irving, P.; Troxler, L.; Galiana-Arnoux, D.; Hetru, C.; Hoffmann, J.A.; Imler, J.L. The Jak-STAT signaling pathway is required but not sufficient for the antiviral response of drosophila. Nat. Immunol. 2005, 6, 946-953. 
105. Waldock, J.; Olson, K.E.; Christophides, G.K. Anopheles gambiae antiviral immune response to systemic O’nyong-nyong infection. PLoS Negl. Trop. Dis. 2012, 6, e1565.

106. Yan, R.; Small, S.; Desplan, C.; Dearolf, C.R.; Darnell, J.E., Jr. Identification of a Stat gene that functions in Drosophila development. Cell 1996, 84, 421-430.

107. Brown, S.; Hu, N.; Hombria, J.C. Identification of the first invertebrate interleukin JAK/STAT receptor, the Drosophila gene domeless. Curr. Biol. 2001, 11, 1700-1705.

108. Binari, R.; Perrimon, N. Stripe-specific regulation of pair-rule genes by hopscotch, a putative Jak family tyrosine kinase in Drosophila. Genes Dev. 1994, 8, 300-312.

109. Kemp, C.; Mueller, S.; Goto, A.; Barbier, V.; Paro, S.; Bonnay, F.; Dostert, C.; Troxler, L.; Hetru, C.; Meignin, C.; et al. Broad RNA interference-mediated antiviral immunity and virus-specific inducible responses in Drosophila. J. Immunol. 2013, 190, 650-658.

110. Souza-Neto, J.A.; Sim, S.; Dimopoulos, G. An evolutionary conserved function of the JAK-STAT pathway in anti-dengue defense. Proc. Natl. Acad. Sci. USA 2009, 106, 17841-17846.

111. Popham, H.J.; Shelby, K.S.; Brandt, S.L.; Coudron, T.A. Potent virucidal activity in larval Heliothis virescens plasma against Helicoverpa zea single capsid nucleopolyhedrovirus. J. Gen. Virol. 2004, 85, 2255-2261.

112. Shelby, K.S.; Popham, H.J. Plasma phenoloxidase of the larval tobacco budworm, Heliothis virescens, is virucidal. J. Insect Sci. 2006, 6, 1-12.

113. Zhao, P.; Lu, Z.; Strand, M.R.; Jiang, H. Antiviral, anti-parasitic, and cytotoxic effects of 5,6-dihydroxyindole (DHI), a reactive compound generated by phenoloxidase during insect immune response. Insect Biochem. Mol. Biol. 2011, 41, 645-652.

114. Tamang, D.; Tseng, S.M.; Huang, C.Y.; Tsao, I.Y.; Chou, S.Z.; Higgs, S.; Christensen, B.M.; Chen, C.C. The use of a double subgenomic Sindbis virus expression system to study mosquito gene function: Effects of antisense nucleotide number and duration of viral infection on gene silencing efficiency. Insect Mol. Biol. 2004, 13, 595-602.

115. Rodriguez-Andres, J.; Rani, S.; Varjak, M.; Chase-Topping, M.E.; Beck, M.H.; Ferguson, M.C.; Schnettler, E.; Fragkoudis, R.; Barry, G.; et al. Phenoloxidase activity acts as a mosquito innate immune response against infection with Semliki Forest virus. PLoS Pathog. 2012, 8, e1002977.

116. Gutierrez, M.G.; Master, S.S.; Singh, S.B.; Taylor, G.A.; Colombo, M.I.; Deretic, V. Autophagy is a defense mechanism inhibiting BCG and Mycobacterium tuberculosis survival in infected macrophages. Cell 2004, 119, 753-766.

117. Ling, Y.M.; Shaw, M.H.; Ayala, C.; Coppens, I.; Taylor, G.A.; Ferguson, D.J.; Yap, G.S. Vacuolar and plasma membrane stripping and autophagic elimination of Toxoplasma gondii in primed effector macrophages. J. Exp. Med. 2006, 203, 2063-2071.

118. Kudchodkar, S.B.; Levine, B. Viruses and autophagy. Rev. Med. Virol. 2009, 19, 359-378.

119. Elmore, S. Apoptosis: A review of programmed cell death. Toxicol. Pathol. 2007, 35, 495-516.

120. Best, S.M. Viral subversion of apoptotic enzymes: Escape from death row. Annu. Rev. Microbiol. 2008, 62, 171-192.

121. Levine, B.; Mizushima, N.; Virgin, H.W. Autophagy in immunity and inflammation. Nature 2011, 469, 323-335.

122. Tanida, I. Autophagy basics. Microbiol. Immunol. 2011, 55, 1-11. 
123. Shelly, S.; Lukinova, N.; Bambina, S.; Berman, A.; Cherry, S. Autophagy is an essential component of Drosophila immunity against vesicular stomatitis virus. Immunity 2009, 30, 588-598.

124. Nakamoto, M.; Moy, R.H.; Xu, J.; Bambina, S.; Yasunaga, A.; Shelly, S.S.; Gold, B.; Cherry, S. Virus recognition by Toll-7 activates antiviral autophagy in Drosophila. Immunity 2012, 36, 658-667.

125. Kerr, J.F.; Wyllie, A.H.; Currie, A.R. Apoptosis: A basic biological phenomenon with wide-ranging implications in tissue kinetics. Br. J. Cancer 1972, 26, 239-257.

126. Fulda, S.; Debatin, K.M. Extrinsic versus intrinsic apoptosis pathways in anticancer chemotherapy. Oncogene 2006, 25, 4798-4811.

127. Courtiade, J.; Pauchet, Y.; Vogel, H.; Heckel, D.G. A comprehensive characterization of the caspase gene family in insects from the order Lepidoptera. BMC Genomics 2011, 12, 357.

128. Settles, E.W.; Friesen, P.D. Flock house virus induces apoptosis by depletion of Drosophila inhibitor-of-apoptosis protein DIAP1. J. Virol. 2008, 82, 1378-1388.

129. Vandergaast, R.; Schultz, K.L.; Cerio, R.J.; Friesen, P.D. Active depletion of host cell inhibitor-of-apoptosis proteins triggers apoptosis upon baculovirus DNA replication. J. Virol. 2011, 85, 8348-8358.

130. Hershberger, P.A.; Dickson, J.A.; Friesen, P.D. Site-specific mutagenesis of the 35-kilodalton protein gene encoded by Autographa californica nuclear polyhedrosis virus: Cell line-specific effects on virus replication. J. Virol. 1992, 66, 5525-5533.

131. Clem, R.J.; Miller, L.K. Apoptosis reduces both the in vitro replication and the in vivo infectivity of a baculovirus. J. Virol. 1993, 67, 3730-3738.

132. Liu, B.; Behura, S.K.; Clem, R.J.; Schneemann, A.; Becnel, J.; Severson, D.W.; Zhou, L. P53-mediated rapid induction of apoptosis conveys resistance to viral infection in Drosophila melanogaster. PLoS Pathog. 2013, 9, e1003137.

133. Karpf, A.R.; Brown, D.T. Comparison of Sindbis virus-induced pathology in mosquito and vertebrate cell cultures. Virology 1998, 240, 193-201.

134. Li, Y.G.; Siripanyaphinyo, U.; Tumkosit, U.; Noranate, N.; A-nueqoonpipat, A.; Tao, R.; Kurosu, T.; Ikuta, K.; Takeda, N.; Anantapreecha, S. Chikungunya virus induces a more moderate cytopathic effect in mosquito cells than in mammalian cells. Intervirology 2013, 56, 6-12.

135. Bowers, D.F.; Coleman, C.G.; Brown, D.T. Sindbis virus-associated pathology in Aedes albopictus (Diptera: Culicidae). J. Med. Entomol. 2003, 40, 698-705.

136. Girard, Y.A.; Schneider, B.S.; McGee, C.E.; Wen, J.; Han, V.C.; Popov, V.; Mason, P.W.; Higgs, S. Salivary gland morphology and virus transmission during long-term cytopathologic West Nile virus infection in Culex mosquitoes. Am. J. Trop. Med. Hyg. 2007, 76, 118-128.

137. Mims, C.A.; Day, M.F.; Marshall, I.D. Cytopathic effect of Semliki Forest virus in the mosquito Aedes aegypti. Am. J. Trop. Med. Hyg. 1966, 15, 775-784.

138. Vaidyanathan, R.; Scott, T.W. Apoptosis in mosquito midgut epithelia associated with West Nile virus infection. Apoptosis 2006, 11, 1643-1651.

139. Weaver, S.C.; Lorenz, L.H.; Scott, T.W. Pathologic changes in the midgut of Culex tarsalis following infection with Western equine encephalomyelitis virus. Am. J. Trop. Med. Hyg. 1992, 47, 691-701.

140. Weaver, S.C.; Scott, T.W.; Lorenz, L.H.; Lerdthusnee, K.; Romoser, W.S. Togavirus-associated pathologic changes in the midgut of a natural mosquito vector. J. Virol. 1988, 62, 2083-2090. 
141. Kelly, E.M.; Moon, D.C.; Bowers, D.F. Apoptosis in mosquito salivary glands: Sindbis virus-associated and tissue homeostasis. J. Gen. Virol. 2012, 93, 2419-2424.

142. Girard, Y.A.; Popov, V.; Wen, J.; Han, V.; Higgs, S. Ultrastructural study of West Nile virus pathogenesis in Culex pipiens quinquefasciatus (Diptera: Culicidae). J. Med. Entomol. 2005, 42, 429-444.

143. Wang, H.; Blair, C.D.; Olson, K.E.; Clem, R.J. Effects of inducing or inhibiting apoptosis on Sindbis virus replication in mosquito cells. J. Gen. Virol. 2008, 89, 2651-2661.

144. Blitvich, B.J.; Blair, C.D.; Kempf, B.J.; Hughes, M.T.; Black, W.C.; Mackie, R.S.; Meredith, C.T.; Beaty, B.J.; Rayms-Keller, A. Developmental- and tissue-specific expression of an inhibitor of apoptosis protein 1 homologue from Aedes triseriatus mosquitoes. Insect Mol. Biol. 2002, 11, 431-442.

145. Ocampo, C.B.; Caicedo, P.A.; Jaramillo, G.; Ursic Bedoya, R.; Baron, O.; Serrato, I.M.; Cooper, D.M.; Lowenberger, C. Differential expression of apoptosis related genes in selected strains of Aedes aegypti with different susceptibilities to dengue virus. PLoS One 2013, 8, e61187.

146. Baron, O.L.; Ursic-Bedoya, R.J.; Lowenberger, C.A.; Ocampo, C.B. Differential gene expression from midguts of refractory and susceptible lines of the mosquito, Aedes aegypti, infected with Dengue-2 virus. J. Insect Sci. 2010, 10, 1-23.

147. Wang, H.; Gort, T.; Boyle, D.L.; Clem, R.J. Effects of manipulating apoptosis on Sindbis virus infection of Aedes aegypti mosquitoes. J. Virol. 2012, 86, 6546-6554.

148. Espada-Murao, L.A.; Morita, K. Delayed cytosolic exposure of Japanese encephalitis virus double-stranded RNA impedes interferon activation and enhances viral dissemination in porcine cells. J. Virol. 2011, 85, 6736-6749.

149. Hastie, K.M.; Kimberlin, C.R.; Zandonatti, M.A.; MacRae, I.J.; Saphire, E.O. Structure of the Lassa virus nucleoprotein reveals a dsRNA-specific $3^{\prime}$ to 5 ' exonuclease activity essential for immune suppression. Proc. Natl. Acad. Sci. USA 2011, 108, 2396-2401.

150. Noyce, R.S.; Taylor, K.; Ciechonska, M.; Collins, S.E.; Duncan, R.; Mossman, K.L. Membrane perturbation elicits an IRF3-dependent, interferon-independent antiviral response. J. Virol. 2011, 85, 10926-10931.

151. Noyce, R.S.; Collins, S.E.; Mossman, K.L. Identification of a novel pathway essential for the immediate-early, interferon-independent antiviral response to enveloped virions. J. Virol. 2006, 80, 226-235.

152. Collins, S.E.; Noyce, R.S.; Mossman, K.L. Innate cellular response to virus particle entry requires IRF3 but not virus replication. J. Virol. 2004, 78, 1706-1717.

153. Rosette, C.; Karin, M. Cytoskeletal control of gene expression: Depolymerization of microtubules activates NF-kappa B. J. Cell Biol. 1995, 128, 1111-1119.

154. Kustermans, G.; el Benna, J.; Piette, J.; Legrand-Poels, S. Perturbation of actin dynamics induces NF-kappaB activation in myelomonocytic cells through an NADPH oxidase-dependent pathway. Biochem. J. 2005, 387, 531-540.

155. Delorme-Axford, E.; Coyne, C.B. The actin cytoskeleton as a barrier to virus infection of polarized epithelial cells. Viruses 2011, 3, 2462-2477.

156. Hare, D.; Mossman, K.L. Novel paradigms of innate immune sensing of viral infections. Cytokine 2013, 63, 219-224. 
157. Gonzalez-Dosal, R.; Horan, K.A.; Rahbek, S.H.; Ichijo, H.; Chen, Z.J.; Mieyal, J.J.; Hartmann, R.; Paludan, S.R. HSV infection induces production of ROS, which potentiate signaling from pattern recognition receptors: Role for S-glutathionylation of TRAF3 and 6. PLoS Pathog. 2011, 7, e1002250.

158. Indukuri, H.; Castro, S.M.; Liao, S.M.; Feeney, L.A.; Dorsch, M.; Coyle, A.J.; Garofalo, R.P.; Brasier, A.R.; Casola, A. Ikkepsilon regulates viral-induced interferon regulatory factor-3 activation via a redox-sensitive pathway. Virology 2006, 353, 155-165.

159. Soucy-Faulkner, A.; Mukawera, E.; Fink, K.; Martel, A.; Jouan, L.; Nzengue, Y.; Lamarre, D.; Vande Velde, C.; Grandvaux, N. Requirement of NOX2 and reactive oxygen species for efficient RIG-I-mediated antiviral response through regulation of MAVS expression. PLoS Pathog. 2010, 6, e1000930.

160. Chen, T.H.; Lo, Y.P.; Yang, C.F.; Chen, W.J. Additive protection by antioxidant and apoptosis-inhibiting effects on mosquito cells with dengue 2 virus infection. PLoS Negl. Trop. Dis. 2012, 6, e1613.

161. Pan, X.; Zhou, G.; Wu, J.; Bian, G.; Lu, P.; Raikhel, A.S.; Xi, Z. Wolbachia induces reactive oxygen species (ROS)-dependent activation of the Toll pathway to control dengue virus in the mosquito Aedes aegypti. Proc. Natl. Acad. Sci. USA 2012, 109, E23-E31.

162. Glaser, R.L.; Meola, M.A. The native Wolbachia endosymbionts of Drosophila melanogaster and Culex quinquefasciatus increase host resistance to West Nile virus infection. PLoS One 2010, 5, e11977.

163. Schroder, M.; Kaufman, R.J. The mammalian unfolded protein response. Annu. Rev. Biochem. 2005, 74, 739-789.

164. Zeng, L.; Liu, Y.P.; Sha, H.; Chen, H.; Qi, L.; Smith, J.A. XBP-1 couples endoplasmic reticulum stress to augmented IFN-beta induction via a cis-acting enhancer in macrophages. J. Immunol. 2010, 185, 2324-2330.

165. Umareddy, I.; Pluquet, O.; Wang, Q.Y.; Vasudevan, S.G.; Chevet, E.; Gu, F. Dengue virus serotype infection specifies the activation of the unfolded protein response. Virol. J. 2007, 4, doi:10.1186/1743-422X-4-91.

166. Patramool, S.; Surasombatpattana, P.; Luplertlop, N.; Seveno, M.; Choumet, V.; Thomas, F.; Misse, D. Proteomic analysis of an Aedes albopictus cell line infected with Dengue serotypes 1 and 3 viruses. Parasit Vectors 2011, 4, doi:10.1186/1756-3305-4-138.

167. Pena, J.; Harris, E. Dengue virus modulates the unfolded protein response in a time-dependent manner. J. Biol. Chem. 2011, 286, 14226-14236.

168. Su, H.L.; Liao, C.L.; Lin, Y.L. Japanese encephalitis virus infection initiates endoplasmic reticulum stress and an unfolded protein response. J. Virol. 2002, 76, 4162-4171.

169. Rathore, A.P.; Ng, M.L.; Vasudevan, S.G. Differential unfolded protein response during Chikungunya and Sindbis virus infection: CHIKV nsP4 suppresses eIF2alpha phosphorylation. Virol. J. 2013, 10, doi:10.1186/1743-422X-10-36.

170. Nene, V.; Wortman, J.R.; Lawson, D.; Haas, B.; Kodira, C.; Tu, Z.J.; Loftus, B.; Xi, Z.; Megy, K.; Grabherr, M.; et al. Genome sequence of Aedes aegypti, a major arbovirus vector. Science 2007, $316,1718-1723$. 
171. Holt, R.A.; Subramanian, G.M.; Halpern, A.; Sutton, G.G.; Charlab, R.; Nusskern, D.R.; Wincker, P.; Clark, A.G.; Ribeiro, J.M.; Wides, R.; et al. The genome sequence of the malaria mosquito Anopheles gambiae. Science 2002, 298, 129-149.

172. Arensburger, P.; Megy, K.; Waterhouse, R.M.; Abrudan, J.; Amedeo, P.; Antelo, B.; Bartholomay, L.; Bidwell, S.; Caler, E.; Camara, F.; et al. Sequencing of Culex quinquefasciatus establishes a platform for mosquito comparative genomics. Science 2010, 330, 86-88.

(C) 2013 by the authors; licensee MDPI, Basel, Switzerland. This article is an open access article distributed under the terms and conditions of the Creative Commons Attribution license (http://creativecommons.org/licenses/by/3.0/). 